\title{
Effectiveness of plant-derived proanthocyanidins on demineralization on enamel and dentin under artificial cariogenic challenge
}

\author{
Ana Paula Pereira da SILVA ${ }^{1}$, Rafael Simões GONÇALVES ${ }^{2}$, Ana Flávia Sanches BORGES ${ }^{2}$, Ana Karina BEDRAN- \\ RUSSO $^{3}$, Mirela Sanae SHINOHARA ${ }^{1}$
}

\footnotetext{
1- Department of Restorative Dentistry, Araçatuba Dental School, UNESP - Univ. Estadual Paulista, Araçatuba, SP, Brazil.

2- Department of Dentistry, Endodontic and Dental Materials, Bauru School of Dentistry, University of São Paulo, Bauru, SP, Brazil.

3- Department of Restorative Dentistry, College of Dentistry, University of Illinois, Chicago, IL, USA.
}

Corresponding author: Prof. Dra. Mirela Sanae Shinohara - Programa de Pós-Graduação em Ciência Odontológica - Área de Concentração: Biomateriais - Faculdade de Odontologia de Araçatuba, UNESP - Univ. Estadual Paulista - R. José Bonifácio, 1193 - Araçatuba - SP - Brazil - 16015-050 - Fax: (+55)18 3636-3349 - E-mail: miodonto@hotmail.com

Submitted: August 14, 2014 - Modification: October 22, 2014 - Accepted: January 22, 2015

\section{ABSTRACT}

\begin{abstract}
$\mathrm{D}$ ental caries is considered a disease of high prevalence and a constant problem in public health. Proanthocyanidins (PAs) are substances that have been the target of recent studies aiming to control or treat caries. Objective: The aim of this in vitro study was to evaluate the efficacy of a treatment with grape seed extract, under cariogenic challenge, to minimize or even prevent the onset of caries in the enamel and dentin. Material and Methods: Blocks of enamel and dentin $(6.0 \times 6.0 \mathrm{~mm})$ were obtained from bovine central incisors, polished, and selected by analysis of surface microhardness (SH). The blocks were randomly divided into 3 groups ( $n=15)$, according to the following treatments: GC (control), GSE (grape seed extract), GF (fluoride $-1,000$ ppm). The blocks were subjected to 6 daily $\mathrm{pH}$ cycles for 8 days. Within the daily cycling, the specimens were stored in buffered solution. The blocks were then analyzed for perpendicular and surface hardness and polarized light microscopy. Results: The means were subjected to statistical analysis using the ANOVA and Fisher's PLSD tests $(p<0.05)$. For enamel SH, GF showed the highest hardness values. In the dentin, GF was also the one that showed higher hardness values, followed by GSE. Regarding the cross-sectional hardness values, all groups behaved similarly in both the enamel and dentin. The samples that were treated with GSE and fluoride (GF) showed statistically higher values than the control. Conclusion: Based on the data obtained in this in vitro study, it is suggested that grape seed extract inhibits demineralization of artificial carious lesions in both the enamel and dentin, but in a different scale in each structure and in a smaller scale when compared to fluoride.
\end{abstract}

Keywords: Dental caries. Proanthocyanidins. Hardness. Dentin. Dental enamel.

\section{INTRODUCTION}

Dental caries is considered a highly prevalent disease that targets a large public ${ }^{11}$. Defined as a multifactorial disease, it results from a dynamic imbalance present in the oral environment in alternating periods of dissolution and mineral replacement ${ }^{12}$, leading to mineral loss.

The demineralization of the dental tissue is caused by acids, especially lactic acid produced by bacterial fermentation of carbohydrates from the diet, usually sucrose. If the acid exposure is short, saliva naturally increases the $\mathrm{pH}$ so that the enamel loss can be repaired by remineralization. However, continuous exposure to acids (ingestion of foods containing sugar) can create a situation in which the remineralization rate may be insufficient to repair the demineralization damage, thereby increasing the development of caries ${ }^{4}$. Therefore, implementing a strategy of protection would be a great aid in a dynamic equilibrium of demineralization and remineralization ${ }^{13}$. 
In recent years, much attention has been focused on research and education related to the identification of food components and development of food products seeking prevention and health promotion ${ }^{28}$. Numerous components that are naturally present in foods and vegetables have been shown to promote health and reduce risk for many common diseases. It is believed that antimicrobial compounds of plant origin can serve as alternatives to the commonly used chemical types for controlling dental plaque and dental diseases ${ }^{28}$.

Proanthocyanidins (PAs) are substances that have been the target of recent studies aiming to control or treat carious lesions ${ }^{29}$. They are naturally presented as a metabolite widely available in fruits, vegetables, nuts, seeds, flowers and bark ${ }^{19}$. Recently, they have been used as natural antioxidants and free radical scavengers, and have been approved in various applications such as clinical and dietary supplements ${ }^{16}$. The PAs present in some fruits prevent the damage caused by reactive oxygen species, inhibiting the glycosyltransferases and possible acid production by Streptococcus mutans ${ }^{1}$. Studies have shown that agents such as glutaraldehyde and extracts rich in PA can be effective in improving the function of the dentin tissue, improving the mechanical stability and reducing collagen degradation ${ }^{6,8}$.

In addition, PA increases the synthesis of collagen, accelerates the conversion of insoluble collagen to soluble collagen during development ${ }^{1}$ and decreases the rate of enzymatic degradation of the collagen matrix ${ }^{27}$. Bedran-Russo, et al. ${ }^{6}$ (2008) showed that a $6.5 \%$ grape seed extract solution could interact with demineralized dentin and increase its modulus of elasticity and strength, due to the stability and increase in the amount of cross-linked collagen ${ }^{21}$. Furthermore, another study about the mandibular bone formation in rats showed that PAs are necessary antioxidants for calcium absorption ${ }^{18}$. In this regard, considering a biomimetic approach using selective natural agents may be a novel approach to stabilize and strengthen the exogenous dentin, inducing collagen crosslinking through interaction with non-collagen protein and by remineralization, possibly decreasing the biodegradation rate and increasing the mineral nucleating ${ }^{5,6}$.

Even with the emergence of new preventive measures, fluoride is still considered one of the most prominent elements because it not only has chemical qualities but physiological properties as well, and is of great interest and importance to the dentist? ${ }^{7}$. Despite its benefits, excess fluoride can be toxic. Its toxicity can be acute or chronic when small amounts are continuously ingested. In its chronic form, the toxicity can affect mineralized tissues (bones and tooth enamel), causing skeletal fluorosis and mainly dental fluorosis.

Thus, this paper analyzed a new treatment that is being studied with the goal of prevention and health promotion, in order to benefit oral health. It verified and tested the hypothesis that grape seed extract, composed mainly of PA, can positively affect the tooth structure, thus offering a new therapy for carious lesions. The null hypotheses were: (1) The application of grape seed extract, rich in PA, has no influence on the enamel submitted to artificial cariogenic challenge and, (2) The application of grape seed extract, rich in PA, has no influence on the dentin submitted to artificial cariogenic challenge.

\section{MATERIAL AND METHODS}

Selection of teeth and specimen preparation fragments of enamel and dentin

Ninety bovine incisors without staining, morphological changes and cracks in the crown were obtained, cleaned and stored in $0.1 \%$ thymol prior to the experiment. The crown of each tooth was separated from the root at the dentin-enamel junction using a diamond disc mounted in an engine bench (Bethil Indústria e Comércio Ltda, Marília, SP, Brazil).

For the enamel analysis, 90 blocks (6.0x6.0 $\mathrm{mm}$ ) were obtained from the buccal surfaces of the crowns by a diamond disk coupled in a metallographic cutter (Isomet 2000, Buehler Ltd., Lake Bluff, IL, USA). For the dentin, 60 root blocks of $6.0 \times 6.0 \mathrm{~mm}$ were obtained (Figure $1 \mathrm{~A}$ ). The surface blocks were then worn and polished in water cooled polishing machine (Aropol 2V Arotec S/A Indústria e. Comércio, Cotia, SP, Brazil) with silicon carbide (SiC) sandpaper in 400, 600 and 1200 grits, followed by polishing pastes of $1.0 \mu \mathrm{m}$ and later $0.5 \mu \mathrm{m}$ (Extec Corp., Enfield, CT, USA) (Figure 1B). The samples were cleaned in ultrasound (Unique, Ultrasonic Cleaner, Indaiatuba, SP, Brazil) at each change of sandpaper with distilled water to remove the residue.

\section{Selection of samples}

After obtaining a flat and polished surface, the initial surface microhardness (HMV-2000 Shimadzu, Columbia, MD, USA) was measured in the center of each specimen. Three rows of five indentations were made with a spacing of $100 \mu \mathrm{m}$ between them, using a load of 25 grams for 15 seconds on the enamel, and a load of 10 grams in the dentin for 10 seconds (Figure $1 \mathrm{C}$ ). This initial measurement allowed the selection of 45 enamel blocks and 45 dentin blocks with hardness values between 320$380 \mathrm{KHN}$, and 30-60 KHN, respectively. 


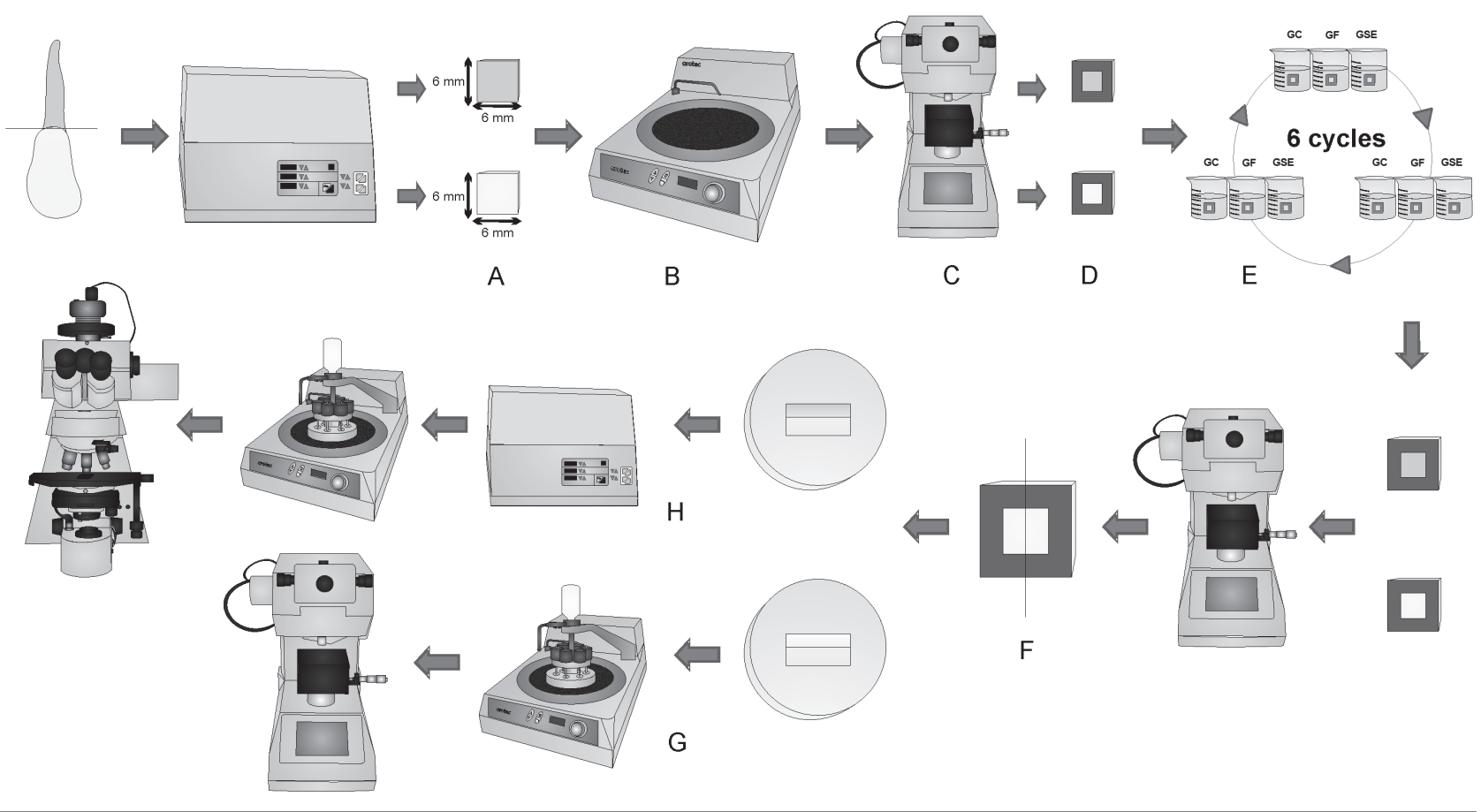

Figure 1- Schematic diagram of the methodology - (A) Obtaining fragments of enamel and dentin; (B) Polishing the specimens; (C) Measuring the initial microhardness; (D) Application of two layers of acid-resistant varnish; (E) $\mathrm{pH}$ cycling with treatments; (F) Sectioning the specimens; (G) Procedures for analysis of the perpendicular hardness to the demineralized surface; $(\mathrm{H})$ Procedures for cross-sectional hardness analysis of the lesion

\section{Experimental groups and preparation of solutions \\ Subsequently, specimens were randomly} divided into three groups ( $n=15)$ according to the treatment: 1) CG - control (no treatment), 2) GSE Grape Seed Extract and 3) GF - fluoride - 1000 ppm ( $\mathrm{NaF}$ solution). Prior to treatment, the fragments received two coats of acid resistant varnish (COLORAMA - L'Oréal Cosmetics Commercial Ltda, São Paulo, SP, Brazil), leaving an area of 4.0 $\mathrm{mm}^{2}$ surface (Figure 1D) for direct contact with the treatments and with the cariogenic challenge (Figure 1E).

The $6.5 \%$ grape seed extract was prepared daily for cycling with deionized water and the $\mathrm{pH}$ adjusted to $7.4^{8,29}$. The demineralizing solution (50 $\mathrm{mM}$ acetate, $2.25 \mathrm{mM} \mathrm{CaCl} 2 \mathrm{H}_{2} \mathrm{O}, 1.35 \mathrm{mM} \mathrm{KH}_{2} \mathrm{PO}_{4}$; $130 \mathrm{~mm} \mathrm{KCl}$ for $\mathrm{pH}=5.0$ ) and the buffer solution (20 mM HEPES, $2.25 \mathrm{mM} \mathrm{CaCl} 2 \mathrm{H}_{2} \mathrm{O}, 1.35 \mathrm{mM} \mathrm{KH}_{2} \mathrm{PO}_{4}$; $130 \mathrm{~mm} \mathrm{KCl}$ for a $\mathrm{pH}=7.0)^{29}$ were prepared on the first day of cycling.

\section{pH cycling (Treatment/Demineralization/ Remineralization)}

The samples were subjected to $\mathrm{pH}$-cycling (demineralization and remineralization) ${ }^{29}$ in conjunction with the treatments according to the groups. Initially, the fragments were placed in
$50 \mathrm{ml}$ of treatment solution (CG, GF, GSE) for 10 minutes, then in $50 \mathrm{ml}$ of demineralizing solution for 30 minutes and finally in $50 \mathrm{ml}$ of buffered solution for 10 minutes (Figure 1F). All the solutions were in constant agitation. These $\mathrm{pH}$ cycles/treatments were performed 6 times a day for 8 days. Between the treatment and $\mathrm{pH}$ cycling, the specimens were washed thoroughly with distilled water. Between the daily cycling, the specimens were stored in buffered solution at $37^{\circ} \mathrm{C}$.

\section{Final surface hardness evaluation}

Following completion of the $\mathrm{pH}$-cycling/treatment process, the samples were placed in ultrasound (Unique, Ultrasonic Cleaner, Indaiatuba, SP, Brazil) for cleaning any remaining residue on their surfaces. Then, the microhardness measurement of the surface was taken (HMV-2000 Shimadzu, Columbia, MD, USA) (Figure 1G). This measurement would be made between the initial hardness indentations; however, due to the process of $\mathrm{pH}$-cycling/treatment in some groups, it was not possible to visualize the initial indentations. Thus, for the final indentations to not be made in the same place as the initial ones (central block), the markings were moved $100 \mu \mathrm{m}$ to the side of the block. The loads used were the same as the initial ones ( 25 grams for 15 seconds on the enamel, and 
10 grams for 10 seconds on the dentin).

At the conclusion of the final surface measurement, the blocks were cut in half and embedded (Figure $1 \mathrm{H}$ ), so the analysis of the crosssectional hardness of the lesion area and Polarized Light Microscopy analysis could be performed.

\section{Cross-sectional hardness evaluation}

For the cross-sectional hardness analysis, the halves were embedded in thermopolymerized acrylic resin (Jet Acrylic Resin - Classic, Campo Limpo Paulista, SP, Brazil) using an embedder (Arotec Pre 30Mi, Arotec SA Ind. and Com., Cotia, $\mathrm{SP}, \mathrm{Brazil})$. The embedded specimens were polished in a water-cooled polishing machine with wet silicon carbide sandpaper in 400, 600 and 1200 grits, followed by polishing pastes of $1.0 \mu \mathrm{m}$ and 0.5 $\mu \mathrm{m}$, respectively. Between each sanding and each paste, the samples were cleaned in ultrasound with distilled water for $5 \mathrm{~min}$ to remove any residue. Transversal microhardness measurements (HMV2000 Shimadzu, Columbia, MD, USA) perpendicular to the demineralized surface were carried out from the surface $(20,50,80,110,140,170$ and 200 $\mu \mathrm{m}$ ) using a load of 5 grams per 10 seconds on the enamel and 10 grams for 10 seconds on the dentin. The measurements were made at three different sites at each depth for all samples (Figure 1G).

After the superficial and cross-sectional hardness analysis, data were tabulated and the normality and homogeneity of variance assumptions were verified. Parametric tests were performed using one-way ANOVA and Fischer's PLSD, using the statistical software Stat View, at a significance level of 0.05.

\section{Polarized light microscopy}

The embedded specimens were then sectioned longitudinally in a metallographic cutter (Isomet 2000, Buehler Ltd., Lake Bluff, IL, USA) to obtain a thickness of around $250 \mu \mathrm{m}$. The histological sections were polished manually with silicon carbide abrasives in 400, 600 and 1200 grits to achieve a thickness close to $100 \mu \mathrm{m}$. The analysis was performed using a polarized light microscope (Axiophot, Zeiss DSM 940 A, Oberkochen, BW, Germany) with a $400 x$ objective. The images of the lesion were qualitatively analyzed between treatments within each substrate (enamel and dentin).

\section{RESULTS}

\section{Enamel}

On the microhardness of the enamel surface, GF showed higher hardness values compared to the other two groups (GC and GSE) $(p<0.0001)$, with no statistical difference between GC and GSE $(p=0.272)$ (Figure 2). Regarding the cross-sectional hardness values, GF and GSE showed statistically higher values than GC, and GF showed the best results (Figure 3 ). All groups showed statistical difference between them $(p<0.0001)$.

In the polarized light microscopic analysis, all the specimens showed areas of negative birefringence, with areas of demineralization in all of them, but they were more pronounced in GC and GSE, indicating that fluoride had a greater preventive potential on enamel than the grape seed extract (Figure 4).

\section{Dentin}

In the dentin, GF also showed the highest values of hardness in relation to the other groups $(p<0.0001)$. There was statistical difference

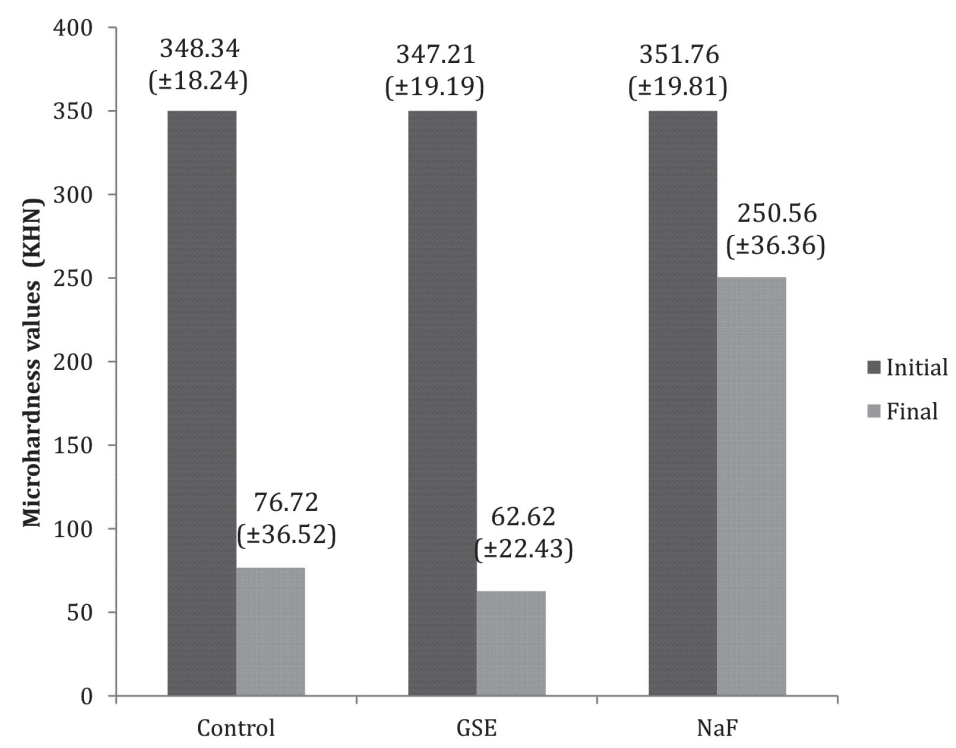

Figure 2- Means and standard deviation of enamel surface hardness (KHN) according to the different groups before and after treatment with fluorides and grape seed extract 
between GSE and the GC, in which GSE showed higher values than GC $(p=0.0009)$ (Figure 5). Regarding the cross-sectional hardness values for the enamel, all groups behaved similarly. The samples treated with grape seed extract and fluoride showed statistically higher values than the control, while GF was statistically higher than GSE
(Figure 6) $(p<0.0001)$

In the analysis of polarized light microscopy, all specimens, as well as the enamel, also had negative birefringence areas showing areas of demineralization, more pronounced in GC and GSE than in GF (Figure 7).

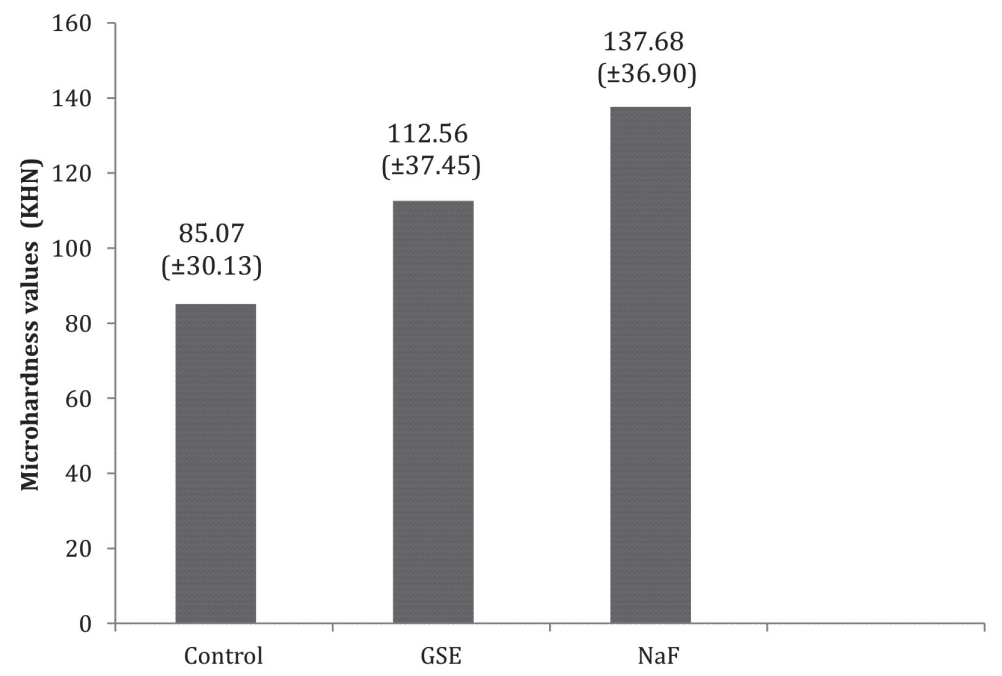

Figure 3- Means and standard deviation of enamel cross-sectional hardness (KHN) according to the different groups after treatment with fluorides and grape seed extract

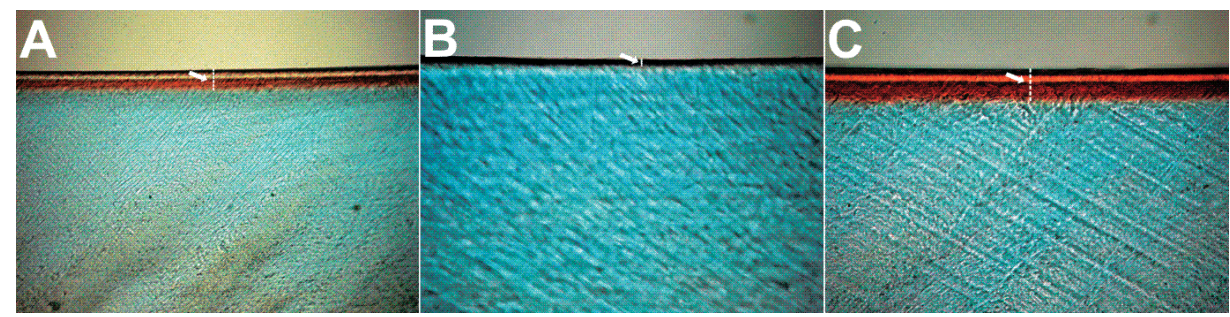

Figure 4- Polarized light microscopy photomicrographs (40x) of enamel lesions in each group after submitted to an artificial cariogenic challenge. Artificial carious lesions - areas of demineralization (arrows: negative birefringence areas); A-control group; B- 1000 ppm NaF; C- GSE

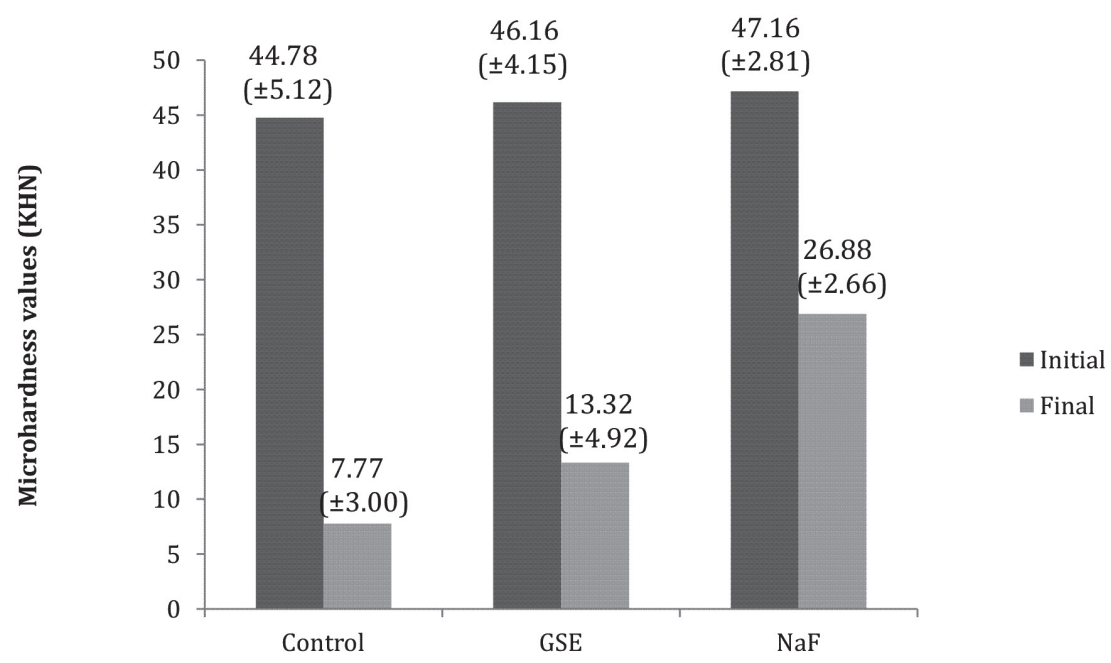

Figure 5- Means and standard deviation of dentin surface hardness (KHN) according to the different groups before and after treatment with fluorides and grape seed extract 


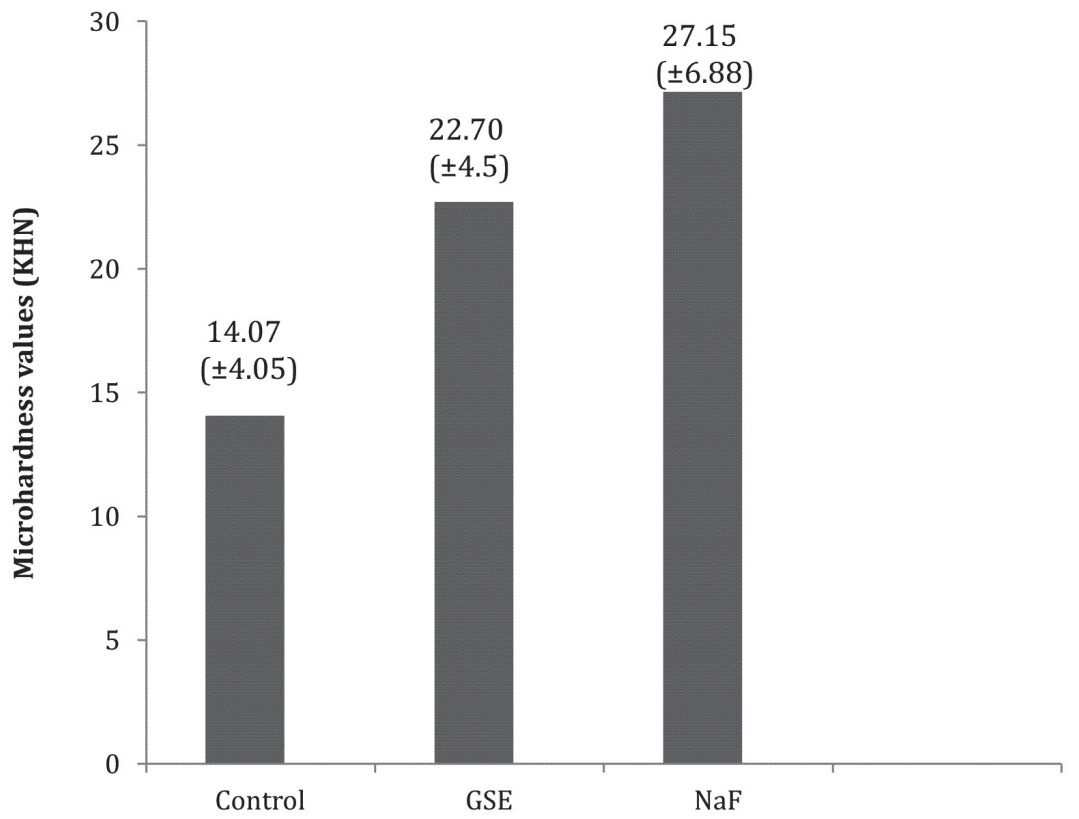

Figure 6- Means and standard deviation of dentin cross-sectional hardness (KHN) according to the different groups after treatment with fluorides and grape seed extract

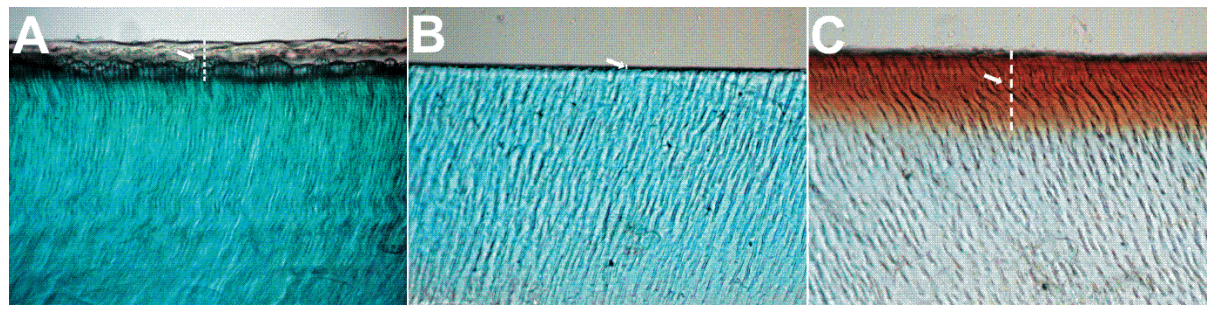

Figure 7- Polarized light microscopy photomicrographs (400x) of dentin lesions in each group after submitted to an artificial cariogenic challenge. Artificial carious lesions - areas of demineralization and staining (red) from grape seed extracted (arrows: negative birefringence areas); A- control group; B- 1000 ppm NaF; C- GSE

\section{DISCUSSION}

Bovine teeth have been widely used in in vitro studies $^{22}$. The in vitro models of $\mathrm{pH}$-cycling are used to simulate the dynamic range of the deposition of minerals occurring in the natural process of carious lesions $^{29}$. This present research was based on the methodology proposed by Xie, Bedran-Russo and $W^{29}$ (2008) and allowed to evaluate the effects of grape seed extract in comparison with fluoride to prevent demineralization in the enamel and dentin using an artificial caries model.

Disease progression or reversal of dental caries depends on the balance between demineralization and remineralization. This balance depends on several factors, such as salivary calcium, phosphate concentration, the bioavailability of fluoride, and the $\mathrm{pH}$ of the saliva ${ }^{2}$.

In present times, natural products have been the focus of much research, to be used as new therapeutic agents ${ }^{15}$. The GSE, used in this study, is composed mainly of PAs, considered powerful antioxidants and known to have vasodilatory effects as well as anti-inflammatory, anti-bacterial and anti-cancer effects ${ }^{24}$.

The PAs represent a variety of flavan-3-ol (catechin) ${ }^{3}$, which is a free radical scavenger required for the absorption of calcium ${ }^{18}$. It is also a polyphenolic compound, and has the potential to give rise to stable hydrogen, and generate non-biodegradable collagen matrices ${ }^{20}$. For these characteristics, GSE has been investigated in the process of the formation of carious lesions.

Enamel demineralization leads to the dissolution of hydroxyapatite and diffusion of calcium and phosphate ions in the direction of its surface ${ }^{25}$. One of the main reasons for enamel demineralization is undoubtedly a decrease in $\mathrm{pH}$ below the critical point that leads to the dissolution of hydroxylapatite ${ }^{14}$. It is well documented that fluoride compounds have been widely used to decrease the development or progression of caries because of its ability to promote remineralization, and reduce the rate of demineralization of teeth ${ }^{7,26}$.

The first null hypothesis was rejected. While GSE has behaved the same as the control in surface 
hardness, in the depth, the grape seed extract reduced the progression of lesions. These results are in agreement with the studies published by Zhao, et al. ${ }^{30}$ (2014) and Mikarimi, et al.23 (2013), which showed that grape seed extract can increase the remineralization of carious enamel lesions, for it could be an effective natural agent for noninvasive dentistry. As its effect on the enamel is not well known, it is possible that the remineralization of this substrate is due to the precipitation of minerals, especially in its interior, since most of its structure is composed of inorganic matter ${ }^{23}$. Moreover, according to Cheng, et al. ${ }^{10}$ (2010), the gallic acid, a major constituent of the grape seed extract, facilitates mineral deposition.

When analyzing the images of polarized light, it was noted that there was lesion formation in all groups, more pronounced in GC and GSE, but according to the cross-sectional hardness values, the structure destruction was higher in GC, showing that the extract had a better potential in depth but smaller in comparison with GF. According to Xie, Bedran-Russo and $\mathrm{Wu}^{29}$ (2008), probably after treatment with the grape seed extract, it is combined with the $\mathrm{Ca}^{+2}$ from the remineralizing solution and may enhance remineralization, but there are still no studies reporting on the activity of the GSE in enamel; thus, more research is needed.

Regarding the process of root caries, this involves two steps: in addition to the dissolution of hydroxyapatite by acid challenge, as in the enamel, there is also the degradation of the organic matrix by proteases of the dentin, saliva, or bacterias ${ }^{9}$. It is well known that the structure and the stability of the collagen matrix are essential for its correct mineralization ${ }^{17}$.

The second null hypothesis was also rejected. In the dentin, GF and GSE also present effectiveness in slowing the progression of carious lesions, and GF also behaved better. According to the data obtained in this study, GSE can positively affect the remineralization process, also by means of mineral deposition, and it can also interact with the organic portion of the root canal by the PA-collagen interaction, stabilizing the exposed collagen matrix. In this way, Bedran-Russo, et al. ${ }^{5}$ (2011), after using grape seed extract in carious dentin, showed an increase in their mechanical properties. Thus, their study suggests that affecting the biochemistry of collagen and proteoglycan interaction, GSE can increase preventive and restorative ability of dentin ${ }^{29}$. Other studies have also shown that GSE can have a positive effect on the remineralization of root caries and dentinal strengthening process. The links between the PAs and proteins have been suggested to involve covalent, ionic, hydrogen or hydrophobic bonds ${ }^{5,12}$.

Analyzing the polarized light images and comparing them with the hardness values, there was lesion formation in all groups and it was more pronounced in GC and GSE, with greater destruction of the structure in the GC. Despite its pronounced area, GSE exerted action on the surface and in depth, but lower than GF.

According to the data obtained in this in vitro study, it is possible to infer that the grape seed extract can inhibit demineralization and/or promote remineralization of carious lesions in the enamel and dentin, showing greater effectiveness in the dentin due to its large acting potential on the links of the collagen matrix. The remineralization effect of the extract appears to be distinct from the action of fluoride. However, the grape seed extract may serve as a promising alternative for the treatment of carious lesions.

\section{CONCLUSION}

Considering the results of this in vitro study, it is believed that the grape seed extract inhibits demineralization of artificial carious lesions in both the enamel and dentin, but in a different way in each structure and in a smaller scale when compared to fluoride.

\section{ACKNOWLEDGMENTS}

The authors wish to thank the São Paulo Research Foundation (FAPESP) for the support (process no. 2012/03396-0). The authors are also grateful to Marjorie de Oliveira Gallinari for the design of chart flow.

\section{REFERENCES}

1- Aldini G, Carini M, Piccoli A, Rossoni G, Facino RM. Procyanidins from grape seeds protect endothelial cells from peroxynitrite damage and enhance endothelium-dependent relaxation in human artery: new evidences for cardio-protection. Life Sci. 2003;73:2883-98.

2- Aoba T. Solubility properties of human tooth mineral and pathogenesis of dental caries. Oral Dis. 2004;10:249-57.

3- Aron PM, Kennedy JA. Flavan-3-ols: nature, occurrence and biological activity. Mol Nutr Food Res. 2008;52:79-104.

4- Aspiras $M$, Stoodley $P$, Nistico $L$, Longwell $M$, de Jager $M$. Clinical implications of power toothbrushing on fluoride delivery: effects on biofilm plaque metabolism and physiology. Int J Dent. $2010 ; 2010: 651869$.

5- Bedran-Russo AK, Castellan CS, Shinohara MS, Hassan L, Antunes A. Characterization of biomodified dentin matrices for potential preventive and reparative therapies. Acta Biomater. 2011;7:1735-41.

6- Bedran-Russo AK, Pashley DH, Agee K, Drummond JL, Miescke $\mathrm{KJ}$. Changes in stiffness of demineralized dentin following application of collagen crosslinkers. J Biomed Mater Res B Appl Biomater. 2008;86:330-4.

7- Cardoso CA, Lacerda B, Mangueira DF, Charone S, Olympio $K P$, Magalhães AC, et al. Mechanisms of action of fluoridated acidic liquid dentifrices against dental caries. Arch Oral Biol. 2014;60:23-8. 
8- Castellan CS, Pereira PN, Grande RH, Bedran-Russo AK. Mechanical characterization of proanthocyanidin-dentin matrix interaction. Dent Mater. 2010;26:968-73.

9- Chaussain-Miller C, Fioretti F, Goldberg M, Menashi S. The role of matrix metalloproteinases (mmps) in human caries. J Dent Res. 2006;85:22-32.

10- Cheng L, Li J, Hao Y, Zhou X. Effect of compounds of Galla chinensis on remineralization of enamel surface in vitro. Arch Oral Biol. 2010;55:435-40.

11- Cochrane NJ, Cai F, Huq NL, Burrow MF, Reynolds EC. New approaches to enhanced remineralization of tooth enamel. J Dent Res. 2010;89:1187-97.

12- Delbem AC, Bergamaschi M, Sassaki KT, Cunha RF. Effect of fluoridated varnish and silver diamine fluoride solution on ename demineralization: pH-cycling study. J Appl Oral Sci. 2006;14:8892.

13- Featherstone JD. Caries prevention and reversal based on the caries balance. Pediatr Dent. 2006;28:128-32.

14- Fejerskov $\mathrm{O}$. Concepts of dental caries and their consequences for understanding the disease. Community Dent Oral Epidemiol. 1997;25:5-12.

15- Ferrazzano GF, Amato I, Ingenito A, Zarrelli A, Pinto G, Pollio A. Plant polyphenols and their anti-cariogenic properties: a review. Molecules. 2011;16:1486-507.

16- Fujii H, Sun B, Nishioka H, Hirose A, Aruoma OI. Evaluation of the safety and toxicity of the oligomerized polyphenol oligonol. Food Chem Toxicol. 2007;45:378-87.

17- Hara AT, Ando M, Cury JA, Serra MC, González-Cabezas C, Zero DT. Influence of the organic matrix on root dentine erosion by citric acid. Caries Res. 2005;39:134-8.

18- Ishikawa M, Maki K, Tofani I, Kimura K, Kimura M. Grape seed proanthocyanidins extract promotes bone formation in rat's mandibular condyle. Eur J Oral Sci. 2005;113:47-52.

19- Joshi SS, Kuszynski CA, Bagchi D. The cellular and molecular basis of health benefits of grape seed proanthocyanidin extract. Curr Pharm Biotechnol. 2001;2:187-200.
20- Kim SJ, Lim MH, Chun IK, Won YH. Effects of flavonoids of Ginkgo biloba on proliferation of human skin fibroblast. Skin Pharmacol. 1997;10:200-05.

21- Macedo GV, Yamauchi M, Bedran-Russo AK. Effects of chemical cross-linkers on caries-affected dentin bonding. J Dent Res. 2009;88:1096-100.

22- Mellberg JR. Hard-tissue substrates for evaluation of cariogenic and anti-cariogenic activity in situ. J Dent Res. 1992;71:913-9. 23- Mirkarimi M, Eskandarion S, Bargrizan M, Delazar A, Kharazifard MJ. Remineralization of artificial caries in primary teeth by grape seed extract: an in vitro study. J Dent Res Dent Clin Dent Prospects. 2013;7:206-10.

24- Rice-Evans CA, Miller NJ. Antioxidant activities of flavonoids as bioactive components of food. Biochem Soc Trans. 1996;24:790-5. 25- Ten Cate JM. Fluorides in caries prevention and control: empiricism or science. Caries Res. 2004;38:254-7.

26- Ten Cate JM, Duijsters PP. Influence of fluoride in solution on tooth demineralization. I. Chemical data. Caries Res. 1983;17:193-9.

27- Walter R, Miguez PA, Arnold RR, Pereira PN, Duarte WR, Yamauchi M. Effects of natural cross-linkers on the stability of dentin collagen and the inhibition of root caries in vitro. Caries Res. 2008;42:263-8.

28- Wu CD. Grape products and oral health. J Nutr. 2009;139:1818S-23S.

29- Xie Q, Bedran-Russo AK, Wu CD. In vitro remineralization effects of grape seed extract on artificial root caries. J Dent. 2008;36:900-6.

30- Zhao W, Xie Q, Bedran-Russo AK, Pan S, Ling J, Wu CD. The preventive effect of grape seed extract on artificial enamel caries progression in a microbial biofilm-induced caries model. J Dent. 2014;42:1010-8. 